\title{
IMPROVEMENT AND CHALLENGES IN QUALITY OF EDUCATION OF PRIMARY AND SECONDARY SCHOOLS IN LALITPUR DISTRICT
}

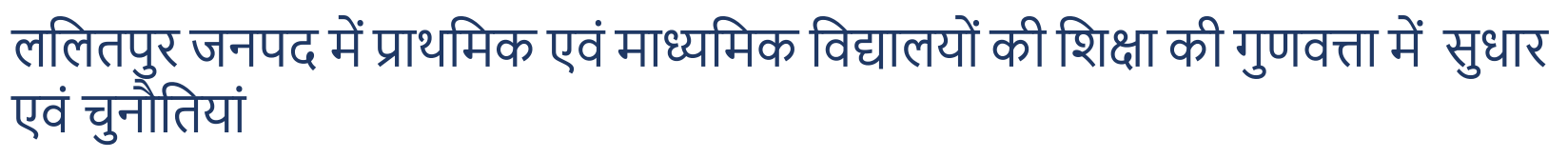

Dr. N.K. Sharma ${ }^{1 凶}$, Mr. Rajesh Kumar Yadav ${ }^{1}$

${ }^{1}$ Adinath College of Education Lalitpur (B.D. Department), India

DOI: https://doi.org/10.29121/granthaalayah.v8.i12.2020.2700

Article Type: Research Article

Article Citation: Dr. N.K. Sharma, Mr. Rajesh Kumar Yadav. (2020). E IMPROVEMENT AND CHALLENGES IN QUALITY OF EDUCATION OF PRIMARY AND SECONDARY SCHOOLS IN LALITPUR DISTRICT. International Journal of Research GRANTHAALAYAH, 8(12), 167-171. https://doi.org/10.29121/granthaalayah.v8.i12.2020.2700

Received Date: 07 December 2020

Accepted Date: 31 December 2020

\section{ABSTRACT}

English: The research study area presented under the title "Improvement and quality of education in primary and secondary school in Lalitpur district". The global economy of the 21st century can thrive in an environment that is based on creativity and imagination, critical thinking and problem-solving skills. There is a strong positive relationship between empirical analysis, economic progress and education. According to the 2011 census in India, school-aged children between the ages of 6-14 years have a huge population of 30.5 million, which is about 25 percent of the total population. The goal of development is to focus on the quality of primary and secondary education by 2030. The Prime Minister of India, Shri Narendra Modi has said in one of his speeches (Mann Ki Baat) about the importance of quality. "Till now the focus of the government was the spread of education across the country but now the time has come to focus on the quality of education Should be given and now attention should be given to schooling knowledge." In this regard, Human Resources Minister Prakash Javadekar has said that" Top priority to improve the quality of education in the country, primary and secondary education facing challenges, 16 percent of 19.67 crore children in 14.5 lakh primary schools under the Sarva Shiksha Abhiyan and 16 percent of the schooling at the secondary level. Has decreased. Thus, to improve the quality of education, skill technical, innovation training technology, information technology employment, education development mission and vocational employment education are urgently needed.

Hindi: प्रस्तुत शोध अध्ययन क्षेत्र "ललितपुर जनपद में प्राथमिक एवं माध्यमिक विद्यालय में शिक्षा की गुणवत्ता में सुधार एवं चुनौतियाँ " शीर्षक के अन्तर्गत अध्ययन किया है। 21 वीं शताब्दी की वैश्चिक अर्थव्यवस्था ऐसे वातावरण की उत्रति कर जो रचनात्मकता एवं काल्पनिकता , विवेचनात्मक सोच और समस्या के समाधान से सम्बन्धित कौशल पर आधारित है। अनुभवमूलक विश्लेषण , आर्थिक उन्नति एवं शिक्षा के मध्य सुदढ़ सकारात्मक सम्बन्ध होते है। भारत में 2011 की जनगणना के अनुसार स्कूल जाने वाले बच्चों की आयु 6-14 वर्ष के मध्य 30.5 करोड़ विशाल जनसंख्या है जो कुल जनसंख्या का लगभग 25 प्रतिशत है। विकास का लक्ष्य 2030 तक प्राथमिक एवं माध्यमिक शिक्षा की गुणवत्ता पर ध्यान देने की आवश्यकता है। भारत के प्रधानमंत्री श्री नरेन्द्र मोदी जी ने अपने एक एक

(C) 2020 The Author(s). This is an open access article distributed under the terms of the Creative Commons Attribution License, which permits unrestricted use, distribution, and reproduction in any medium, provided the original author and source are credited. 
उद्वोधन (मन की बात) में गुणवत्ता के महत्व में कहा है " अब तक सरकार का ध्यान देश भर में शिक्षा का प्रसार था किन्तु अब वक्त आ गया है कि ध्यान शिक्षा की गुणवत्ता पर दिया जाय और अब स्कूलिंग ज्ञान पर ध्यान देना चाहिएं।" इस सम्बन्ध में मानव संसाधन मंत्री प्रकाश जावड़ेकर ने कहा है कि " देश में शिक्षा की गुणवत्ता में सुधार की सर्वोच्च प्राथमिकता, चुनौतियों का सामना करके प्राथमिक एवं माध्यमिक शिक्षा, सर्वशिक्षा अभियान के अन्तर्गत 14.5 लाख प्राथमिक विद्यालय में 19.67 करोड़ बच्चों की प्राथमिक स्तर पर 16 प्रतिशत तथा माध्यमिक स्तर पर 32 प्रतिशत बच्चों की स्कूली शिक्षा में कमी हुई है। इस प्रकार शिक्षा की गुणवत्ता को बेहतर बनाने के लिए कौशल तकनीकी, नवाचार प्रशिक्षण तकनीकी, सूचना तकनीकी रोजगार, शिक्षा विकास मिशन तथा व्यावसायिक रोजगार परक शिक्षा की अति आवश्यकता है।

Keywords: ललितपुर; शिक्षा; विद्यालया

\section{1. प्रस्तावना}

अध्ययन क्षेत्र जनपद ललितपुर में प्राथमिक एवं उच्च प्राथमिक विद्यालयों में शिक्षा की गुणवत्ता के स्तर को सुधारने के लिए केन्द्र सरकार एवं राज्य सरकार दोनों सरकारें नवीन व्यापक हृष्कोण एवं रणनीतियों को बना रहे हे, जिससे अध्यापक कक्षा में अपनायी जाने वाली कार्यविधियो, छात्रों में ज्ञान के मूल्यांकन एवं निर्धारण विद्यालयी अवसंरचना , विद्यालयी प्रभावशीलता एवं सामाजिक सहभागिता से सम्बन्धित मुद्दों पर कार्य कर रही है। देश में शिक्षा अधिकार अधिनियम 2009 लागू हुआ तो 6 वर्ष से 14 वर्ष के बच्चों के लिए यह मौलिक अधिकार बन गया। इसके अलावा शिक्षा के क्षेत्र में केन्द्र एवं राज्य सरकार के द्वारा विभित्र योजनाएँ एवं कार्यक्रम चलाये जा रहे है। इसके बावजूद शिक्षा के क्षेत्र में चुनौतियों का अम्बार लगा हुआ है। ऐसे उपायों की तलाश में प्रयास जारी है। मानव संसाधन के विकास का मूल शिक्षा है जो देश के सामाजिक, आर्थिक तन्त्र के सन्तुलन में शिक्षा में सुधार की महत्वपूर्ण भूमिका है।

शिक्षा के क्षेत्र में 1976 में 42 वें संविधान संशोधन अधिनियम के अन्तर्गत शिक्षा की समवर्त्ती सूची का विषय बनाया गया, जिस पर केन्द्र एवं राज्य सरकारें नियम बना सकती है। शिक्षा का अधिकार, बाल शिक्षा का अधिकार (21।) को जोड़कर 2002 में कानून पारित हुआ। मानव संसाधन विकास मंत्रालय ने स्कूली शिक्षा को स्कूल पूर्व प्राइमरी, अपर प्राइमरी, माध्यमिक और उच्च माध्यमिक स्तरों पर बाँटकर गुणवत्ता को बनाये हुए है। राष्ट्रीय शिक्षा मिशन के अन्तर्गत सूचना एवं संचार प्रौद्योगिकी के माध्यम से (R.S.M.) के द्वारा केन्द्र सरकार की योजना है। I.C.T. के माध्यम से विद्यार्थी विषय वस्तु से सम्बन्धित सामग्री से सुलभ ज्ञान प्राप्त करते है। राष्ट्रीय नीति आयोग ने एक राज्यस्तरीय स्कूली शिक्षा की गुणवत्ता सूचकांक (SEQI) बनाया है तथा सभी राज्यों के विद्यालयी शिक्षा गुणवत्ता का मानदण्ड तैयार किया जाता है और रैकिंग ग्रेड के माध्यम से राज्यों को प्रतिस्पर्धा को प्रोत्साहित किया जाता है तथा अन्य राज्यों को सुधार के लिए प्रेरित किया जाता है। अध्ययन क्षेत्र में प्राथमिक एवं माध्यमिक दोनों स्तरों की शिक्षा पर शिक्षकों की क्षमता में बढ़ोत्तरी को गम्भीरतापूर्वक विचार करके जमीनी स्तर पर शिक्षा की गुणवत्ता का बदलाव लाने में शिक्षकों की महत्वपूर्ण भूमिका होगी। सहायक अध्यापक अपने अध्ययन क्षेत्र ललितपुर जनपद में प्राथमिक एवं उच्च प्राथमिक विद्यालयों में कार्यरत छात्र/छात्रों की संख्या एवं विद्यालय की संख्या के आधार पर विश्लेषण किया गया है।

ललितपुर जनपद में प्राथमिक एवं माध्यमिक विद्यालयों में अध्ययनरत छात्र/छात्राओं से सम्बन्धित संख्यायें: 2018-19

\begin{tabular}{|c|c|c|c|c|c|c|c|c|}
\hline क0 & विकासखण्ड का नाम & प्रा0 वि0 & \multicolumn{2}{|c|}{$1-5$ तक कक्षा } & \multicolumn{2}{|c|}{ उच्च प्रा0 वि0 } & \multicolumn{2}{c|}{6 तक कक्षा } \\
\cline { 4 - 8 } सं0 & & की संख्या & छात्र & छात्रायें & छात्र & छात्रायें & छात्र & छात्रायें \\
\hline 1 & तालबहेट & 199 & 11828 & 10652 & 83 & 11 & 6252 & 5881 \\
2 & जखौरा & 251 & 14759 & 13613 & 112 & 16 & 7504 & 7289 \\
3 & बार & 187 & 10930 & 9903 & 86 & 5 & 5374 & 4905 \\
4 & बिरघा & 244 & 13576 & 12697 & 111 & 7 & 6524 & 6344 \\
5 & महरौनी & 186 & 13276 & 11623 & 93 & 8 & 7221 & 6601 \\
6 & मंडावरा & 189 & 10872 & 10272 & 101 & 9 & 5180 & 4798 \\
\hline & ग्रामीण योग & 1256 & 75241 & 68760 & 586 & 56 & 38055 & 35818 \\
\hline & नगरीय योग & 162 & 7390 & 6440 & 57 & 8 & 2635 & 2367 \\
\hline & जनपद योग & 1418 & 82631 & 75200 & 643 & 64 & 40690 & 38185 \\
\hline
\end{tabular}

स्रोतः जनपद सांख्यिकी पत्रिका ललितपुर जनपद 2018-19 तालिका सं0 39,4 
ललितपुर जनपद में प्राथमिक एवं माध्यमिक विद्यालयों की शिक्षा की गुणवत्ता में सुधार एवं चुनौतिया

शोध क्षेत्र ललितपुर जनपद में प्राथमिक एवं उच्च प्राथमिक विद्यालयों में अध्ययनरत छात्रों की संख्या एवं विद्यालयों की संख्या प्राथमिक स्तर (कक्षा 1 से 5 तक) तक ग्रामीण क्षेत्र में 1256 तथा नगरीय क्षेत्र में 162 प्राथमिक विद्यालय है। जनपद में कुल प्राथमिक विद्यालयों की संख्या 2018-19 की जनसांख्यिकीय पत्रिका के अनुसार 1418 है तथा उच्च प्राथमिक विद्यालयों की संख्या ग्रामीण क्षेत्रों में (कक्षा 6 से 8 तक) 586 है तथा नगरीय क्षेत्रों मे 57 है। जनपद में कुल उच्च प्राथमिक विद्यालयों की संख्या 643 हैं जिसमें बालिका उच्च प्राथमिक विद्यालयों की संख्या ग्रामीण स्तर पर 56 है तथा नगरीय स्तर पर 8 है और जनपद स्तर पर 64 उच्च प्राथमिक विद्यालय है। प्राथमिक विद्यालय में कुल ग्रामीण स्तर पर छात्रों की संख्या 75241 है तथा नगरीय स्तर पर 7390 है । जनपद में कुल प्राथमिक विद्यार्थियों की संख्या 82631 छात्र पंजीकृत है जबकि प्राथमिक स्तर छात्राओं की संख्या ग्रामीण स्तर पर 68760 है। । नगरीय स्तर पर 6440 है। जनपद में कुल छात्राओं की संख्या 75200 है। इस प्रकार उच्च प्राथमिक स्तर में (कक्षा 6 से 8 तक) विद्यालयों की संख्या 643 है, जबकि ग्रामीण स्तर पर 586 नगरीय स्तर पर 57 विद्यालय है। कक्षा 6 से 8 तक पंजीकृत छात्रों की संख्या

40690 है तथा छात्राओं की संख्या 38185 है जो लगभग 2018-19 से प्राप्त किया गया है। इस प्रकार से ललितपुर जनपद में प्राथमिक एवं उच्च प्राथमिक विद्यालय शिक्षा की गुणवत्ता का विश्लेषात्मक अध्ययन किया गया है।

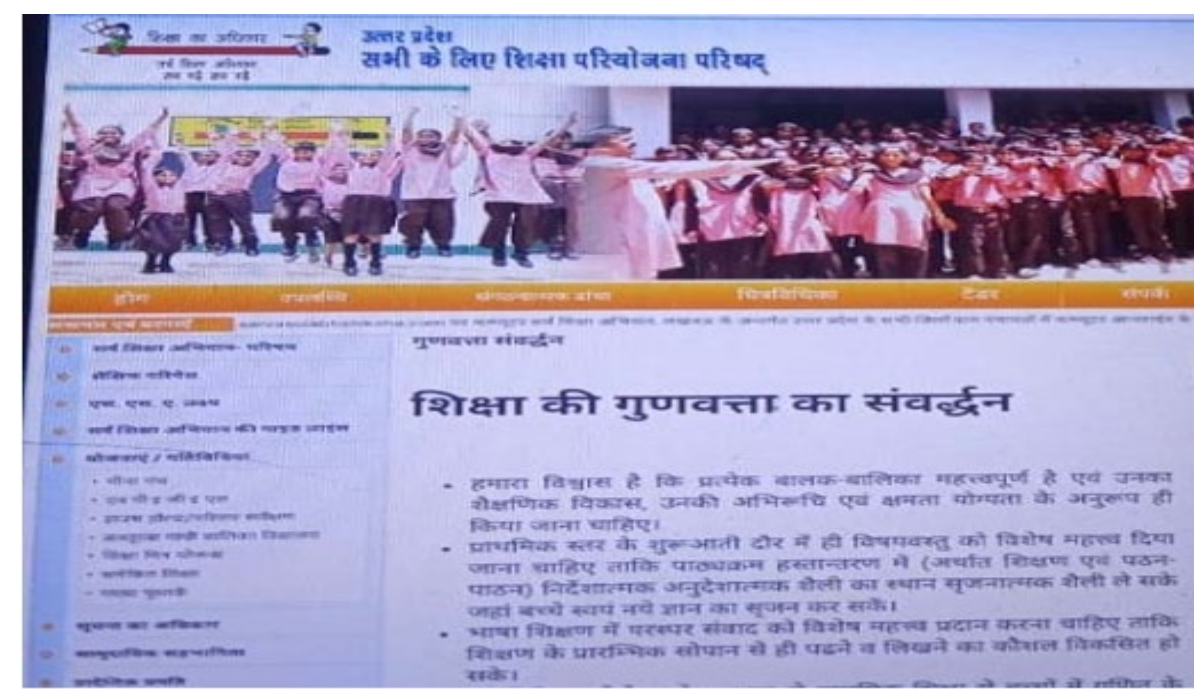

\section{2. ललितपुर जनपद में प्राथमिक एवं उच्च प्राथमिक विद्यालयी शिक्षा की गुणवत्ता में सुधार एवं चुनौतियाँ:}

\section{1. अध्यापक की भूमिका}

बच्चे विद्यालयी शिक्षा के केन्द्र होते हैं। बच्चों के ज्ञानार्जन सुनिश्चित करने में सबसे महत्वपूर्ण भूमिका अध्यापक की होती है। सर्वशिक्षा अभियान की शुरूआत के ही प्रारम्भिक कक्षाओं में अध्यापकों के 19.48 लाख पद प्राथमिक एवं उच्च प्राथमिक पदों का सृजन किया गया है। इन पदों के अध्यापकों की नियुक्ति से छात्र शिक्षक का अनुपात $42: 1$ से $24: 1$ का सुधार हुआ है। वर्तमान समय में सरकारी विद्यालयों में नियमित अध्यापकों में से 85 प्रतिशत व्यावसायिक कार्य में सफल हैं। मंत्रालय द्वारा 2013 की अध्यापक गणना के अनुसार 83 प्रतिशत उपस्थित होती है। इसको बढ़ाकर 100 प्रतिशत लाने की आवश्यकता है। सर्वशिक्षा शिक्षा अभियान एवं राष्ट्रीय साक्षरता मिशन के अध्यापकों के व्यावसायिक विकास के प्रशिक्षण कार्य के माध्यम से सेवारत अध्यापकों को प्रशिक्षित कर प्राथमिक एवं माध्यमिक शिक्षा की गुणवत्ता में सुधार किया जा सकता है , जिसके परिणामस्वरूप विद्यालयी तन्त्र में प्रतिभाशाली युवाओं को अध्यापन के क्षेत्र में राष्ट्रीय शिक्षक शिक्षा परिषद के चार वर्षीय समेकित बी० ए0 बी0 एड0, बी0 एस0सी0 बी0 एड 0 की शुरूआत की है तथा ईमानदारी से रूचि रखने वाले कार्यक्रमों का प्रसार-प्रचार किया जाना चाहिए।

\section{2. नवाचार कौशल तकनीक का प्रयोग}

शिक्षा के क्षेत्र में नवाचार संसाधनों के माध्यम से प्राथमिक एवं उच्च प्राथमिक शिक्षा की गुणवत्ता को सुधारने एवं चुनौतियों का सामना करने में नवाचार (कौशल तकनीक) की महत्वपूर्ण भूमिका होती है। E.T. के माध्यम से कम्प्यूटर, इण्टरनेट, लैपटा प एवं अन्य सूचना संचार प्रौद्योगिकी द्वारा विद्यालयी शिक्षा की गुणवत्ता में सुधार किया जा सकता है। इस प्रकार से शिक्षा के क्षेत्र में कौशल विकास योजना के अन्तर्गत प्रधानमंत्री कौशल विकास एक महत्वपूर्ण भूमिका हर व्यावसायिक कार्यों में प्रगतिशील है। 


\section{3. कक्षा कक्ष में अपनाई जाने वाली कार्यविधियाँ}

अध्ययन क्षेत्र में प्राथमिक एवं उच्च प्राथमिक शिक्षा बच्चों में ज्ञान की समझ को विकसित करने के लिए कक्षा कक्ष प्रबन्धन , प्रभावी छात्र-शिक्षक संवाद, निर्देशों की उत्तमता, संरक्षित अध्ययन एवं सीखने पर जोर देने की गतिविधियों के दृष्टिकोण से इन कार्यविधियों का सर्वाधिक महत्व है। छात्र एवं अध्यापक की कक्षा कक्ष नियमित रूप से उपस्थित पूर्व प्रतिबन्ध हैं। आई0 सी0 टी० समर्थित शिक्षण एवं अधिगम के सन्दर्भ में सीखने की प्रक्रिया के परिणाम पर विशेषरूप से ध्यान दिये जाने की आवश्यकता है। विद्यालय शिक्षकों एवं विद्यालय के माता-पिता के और समुदाय के बीच व्यापक रूप से प्रसारित किया जा सके। इस कार्यक्रम का शुभारम्भ 2014 में “ प पढ़े भारत-बढ़े भारत“ हेतु मजबूत बुनियाद को स्वीकार किया गया है। जिसमें गणित, विज्ञान, प्रौद्योगिकी के अध्ययन को लोकप्रिय बनाया गया है। देश के सम्पूर्ण शिक्षा क्षेत्र में ;छत्क्म्द्ध के अन्तर्गत सभी ई - पाठशाला विद्यालय शिक्षा और शिक्षक को ई-डिजिटल और डिजिटल संसाधनों के द्वारा शिक्षा के क्षेत्र में व्यापक सुधार किया गया है।

\section{4. मूल्यांकन और आँकलन}

अध्ययन क्षेत्र जनपद सहित सभी छात्र की प्रगति का आकलन करने में शिक्षक की महत्वपूर्ण भूमिका होती है। कक्षा में छात्रों के नियमित मूल्यांकन से अभिप्राय बच्चों और माता-पिता को प्रतिक्रिया देना शिक्षक को प्रतिक्रिया और बच्चों के बीच अध्ययन की समस्या के समाधान हेतु प्राथमिक एवं उच्च प्राथमिक विद्यालयों के शिक्षण परिणामों को सुधारने हेतु सकारात्मक प्रयास किया जाना चाहिए । शैक्षणिक व्यवस्था की स्थिति एवं पाठ्यक्रम शैक्षणिक प्रयासों के बीच समन्वय करके शिक्षा के स्तर में सुधार किया जा सकता है।

\section{5. विद्यालय प्रभावशीलता}

अध्ययन क्षेत्र में विद्यालयों को प्रभावी ढंग से प्रदर्शन के लिए विद्यालय प्रमुख का सशक्तिकरण महत्वपूर्ण हैं। भारत सरकार ने राज्य सरकारों को प्रधानाचार्य के लिए एक पृथक कैलेण्डर बनाने के लिए कदम उठाने की सलाह दी है तथा पूर्णकालीन प्रधानाचार्य के क्षमता निर्माण के लिए विद्यालयी शिक्षण प्रशिक्षण को (NUEPA) पर राष्ट्रीय विद्यालय नेतृत्व केन्द्र ने एक प्रशिक्षण पैकेज तैयार किया है। 2016 में विद्यालय प्रभावशीलता कार्यक्रम का शुभारम्भ किया गया है। छात्रों एवं शिक्षकों के मध्य सभी पात्र बच्चे मध्याह्न भोजन , पाठ्य पुस्तके और छात्रवृत्तियों को प्राप्त करने के साथ-साथ शिक्षक छात्र उपस्थिति की निगरानी विद्यालय प्रभावशीलता के मूल्यांकन में महत्वपूर्ण है।

\section{6. विद्यालय बुनियादी ढांचा}

सर्वशिक्षा अभियान और राष्ट्रीय शिक्षा मिशन के अन्तर्गत विभित्र हस्तक्षेपों के माध्यम से विद्यालय के बुनियादी ढ़ाँचे के प्रावधानों के तहत उल्लेखनीय प्रगति हुई है। एस 0 एस 0 ए 0 के प्रारम्भ होने के बाद से 2.23 लाख प्रारम्भिक और करीब 4 लाख उच्च प्राथमिक विद्यालयों के लिए विद्यालय भवन तैयार किये गये हैं। प्रत्येक विद्यालय में छात्र छात्रों के लिए एक पृथक कार्यात्मक शौचालय होने के प्रधानमंत्री के आधार सभी राज्यों एवं केन्द्र शासित प्रदेशों में सार्वजनिक एवं निजी संस्थानों में सकारात्मक प्रक्रिया व्यक्त की है तथा स्वच्छ विद्यालय की पहल के अन्तर्गत 4.17 लाख शौचालय का निर्माण कराया गया है। इसके अन्तर्गत बिजली कनेक्शन, एल 0 पी 0 जी 0 गैस, कम्प्यूटर प्रयोगशाला पाठन स्थल निर्माण आदि।

\section{7. सामुदायिक भागीदारी}

अध्ययन क्षेत्र में एक व्यापक और विविधता से भरे देश में निर्णय लेना और जबाबदेही का विकेन्द्रीकरण ही सफलता की कुन्जी हैं। विद्यालय शिक्षा के मामले में समुदाय विद्यालय प्रबन्धन समितियों के माध्यम से विद्यालय प्रबन्धन में सामुदायिक भागीदारी एक महत्वपूर्ण भूमिका मानी जाती है। एम0एम0सी0 बैठक सामाजिक अंकेक्षण अथवा शिक्षा शिक्षण लक्ष्यों पर ग्राम सभा की बैठकों जैसे प्रयासों के फलस्वरूप विद्यार्थी के अध्ययन के जोड़ने और मूल्यांकन में की अति आवश्यकता होगी।

\section{3. निष्कर्ष एवं सुझाव}

अध्ययन क्षेत्र ललितपुर जनपद में प्राथमिक एवं उच्च प्राथमिक विद्यालयी शिक्षा के सुधार एवं चुनौतियों के सम्बन्ध में शिक्षा की सकारात्मक गुणवत्ता की महत्वपूर्ण भूमिका मानी जाती है। प्राथमिक शिक्षा के विकास हेतु 6 से 14 वर्ष के सभी छात्र छात्रों को सर्वशिक्षा मिश के अन्तर्गत गुणात्मक शिक्षेण व्यवस्था की आवश्यकता वर्तमान समय में है। शिक्षा के क्षेत्र में नवीन नवाचार संसाधनों के द्वारा 
ललितपुर जनपद में प्राथमिक एवं माध्यमिक विद्यालयों की शिक्षा की गुणवत्ता में सुधार एवं चुनौतिया

व्यावसायिक रोजगार परक शिक्षा की आवश्यकता उच्च माध्यमिक एवं उच्च प्राथमिक शिक्षा के लिए आवश्यक माना जाता है। प्रधानमंत्री कौशल विकास मिशन के अन्तर्गत उच्च प्राथमिक एवं माध्यमिक शिक्षा स्तर पर सभी क्षेत्रो मे रोजगार परक शिक्षा बढ़ाने की आवश्यकता है। ललितपुर जनपद प्राथमिक एवं माध्यमिक शिक्षा के गुणात्मक सुधार हेतु चुनौतियों का सामना करते हुए अनेक कौशल तकनीकी प्रौद्योगिक के माध्यम से गुणात्मक, ज्ञानात्मक शिक्षा के स्तर में सुधार किया जा रहा है, जिसके परिणामस्वरूप 2030 तक 2 करोड़ लोगों को कौशल विकास मिशन के अन्तर्गत गुणात्मक शिक्षण का प्रसार प्रचार होगा।

\section{SOURCES OF FUNDING}

None.

\section{CONFLICT OF INTEREST}

None.

\section{ACKNOWLEDGMENT}

None.

\section{REFERENCES}

[1] जनपद जनसांख्यिकीय पत्रिका ललितपुर 2018-19 तालिका संख्या 39,40

[2] डॉ. सुबाष सी खुटिया: विद्यालय शिक्षा साक्षरता मिशन 2017 पत्र

[3] सूचना विभाग नई दिल्ली।

[4] पत्र-पत्रिका, समाचार, इण्टरनेट

[5] योजना पत्रिका अगस्त ।

[6] लेख, जर्नल पत्रिका नई दिल्ली

[7] शिक्षा सूचना विभाग लखनऊ, 2018 